\title{
INVESTIGACIONES
}

\section{Problemas en la inserción profesional de profesores: necesidad de mentoría*}

\author{
Problems of the professional insertion of teachers: the need for mentoring
}

\author{
Ma. Cristina Solís Zañartu , Claudio Núñez Vegab, Nelson Vásquez Larac, \\ Inés Contreras Valenzuelad, Sylvia Ritterhaussen Klaunninge

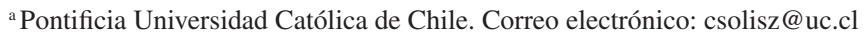 \\ b Pontificia Universidad Católica de Chile. Correo electrónico: cnunezv@uc.cl \\ c Pontificia Universidad Católica de Valparaíso. Correo electrónico: nvasquez@ucv.cl \\ d Pontificia Universidad Católica de Chile. Correo electrónico: icontrev@uc.cl \\ ${ }^{\text {e }}$ Pontificia Universidad Católica de Chile. Correo electrónico: sritters@uc.cl
}

RESUMEN

Este estudio presenta problemas percibidos por profesores novatos de educación básica y media de universidades chilenas en los tres primeros años de inserción profesional.

Se identifican tareas del desempeño consideradas por los profesores principiantes como problemas, los que se mantienen durante este período de ejercicio profesional. De ellos algunos son indicadores de debilidades en la formación inicial y otros de falencias en las condiciones de los centros educativos.

Este estudio visualiza la necesidad de contar con programas de mentoría en la formación práctica y en los primeros años de ejercicio profesional, para disminuir la tensión producida al inicio de la vida laboral.

Estos hallazgos son útiles para las universidades y los centros educativos que contratan profesores novatos, para diseñar y aplicar procesos de mentoría que apoyen el desarrollo profesional de los profesores.

Palabras clave: profesores novatos, problemas en la inserción profesional, necesidad de mentoría

\begin{abstract}
This study presents problems perceived by beginning teachers in elementary and secondary education of Chilean universities and in the first three years of professional insertion. Performance tasks considered by beginning teachers as problems and which are maintained during this period of practice are identified. Some of them are indicative of weaknesses in the initial training and others as shortcomings in the conditions of schools. This study displays the need for mentoring programs and practical training in the early years of practice to decrease the voltage produced at the start of the working life. These findings are useful for universities and schools that hire novice teachers to design and implement mentoring processes to support the professional development of teachers.

Key words: beginning teachers, professional insertion problems, need for mentoring
\end{abstract}




\section{ANTECEDENTES}

El artículo aquí presentado contiene parte de los resultados de una investigación que indaga, en Chile, las condiciones de inserción profesional de los egresados de las carreras de pedagogía media y básica, considerando el desempeño en aula de los profesores novatos, las dificultades vivenciadas, las herramientas con que cuentan para resolver dichas dificultades y los sistemas de apoyo desde las instituciones educativas. La fuente de información estuvo constituida por profesores principiantes de seis instituciones formadoras de profesores chilenas, tanto de educación básica como de educación media, directivos de dichas carreras y directores de los establecimientos en los cuales esos profesores novatos se desempeñaban en el primer año de ejercicio profesional.

Esta investigación implicó la realización de dos tipos de estudio. El primero, de tipo transeccional, que busca conocer la opinión de los profesores novatos con respecto a las dificultades y éxitos durante sus tres primeros años de inserción laboral y la opinión de los directivos de establecimientos en que se desempeñaban, con respecto a los apoyos que la institución ofrece a los profesores novatos en su proceso de inserción laboral. El segundo es un estudio longitudinal que busca indagar respecto de la evolución de los profesores novatos en su desempeño en aula en los primeros tres años de inserción profesional y su percepción de las dificultades que enfrentan a medida que se desarrollan profesionalmente.

Los resultados presentados en este artículo corresponden a ambos estudios, transeccional y longitudinal, los cuales permiten identificar las dificultades percibidas por los profesores principiantes en los tres primeros años de ejercicio profesional. Estos resultados evidencian la necesidad de apoyo requerido por los profesores para iniciar la vida laboral, de los cuales deberían hacerse cargo, por una parte, las instituciones formadoras, en aquellas áreas en que las dificultades derivan de la formación inicial, y por otra, las instituciones educativas en áreas relacionadas con el contexto específico de cada escuela; ambos tipos de apoyo no solo facilitarían la inserción a la vida profesional de los profesores, sino que contribuirían a su desarrollo profesional, desde una realidad situada.

\section{MARCO REFERENCIAL}

La investigación internacional, desde hace más de una década, ha establecido que el inicio de la profesión docente implica un desafío relevante para los profesores jóvenes. Algunos enfrentan con éxito la etapa, realizando innovaciones en el aula, otros se someten rápidamente a las culturas escolares, desarrollando un quehacer rutinario, y un porcentaje no menor es superado por los contextos escolares y desertan de la profesión, con las implicancias que esto tiene para los países.

Los profesores principiantes transitan desde la condición de estudiante hacia la de profesor; esta transición ha sido definida como el proceso de iniciación a la enseñanza. Ellos dejan una institución conocida, en la que tienen pares con los cuales han compartido algunos años y se incorporan a otra institución cuya cultura organizacional desconocen y donde deben asumir nuevas responsabilidades. Esta transición, que también ha sido denominada como un "eslabón perdido", debería aprovecharse para establecer la continuidad entre la formación inicial y la formación continua (Eirin, García y Montero, 2009). 
Al insertarse en los establecimientos escolares, los profesores principiantes suelen enfrentarse a numerosas problemáticas de desigual complejidad, algunas derivadas de las naturales relaciones entre profesionales, otras del escaso conocimiento que tienen de la cultura escolar o de las múltiples tareas a resolver simultáneamente, además de asumir la complejidad de enseñar y lograr aprendizaje en contextos escolares diversos.

La inserción profesional docente ha sido abordada en diferentes estudios nacionales e internacionales. Algunos autores la consideran como una etapa más en el desarrollo profesional, con características propias que van cambiando a medida que el nuevo profesor adquiere los conocimientos disciplinarios, pedagógicos y de los contextos en los que se desenvuelve. Otros sostienen que es un período de transición entre la formación y la vida profesional. Sin embargo todos coinciden en que hay un conjunto de dificultades que el profesor principiante debe sortear durante los primeros años (Marcelo, 2008). Además, este autor plantea que la inserción profesional es el período en el cual los profesores transitan desde estudiantes a maestros, y complementa su visión con los planteamientos de Vonk (1996), quien señala que en este período hay una transición entre ser profesor en formación y profesional autónomo, y que ambas etapas forman parte del continuo del desarrollo profesional, por lo que la inserción profesional es parte del desarrollo profesional.

El éxito del profesor principiante en sus primeros años dependerá de sus capacidades para conocer la institución escolar y sortear las dificultades con que se encuentra, independientemente de la preparación que le ha entregado la institución formadora o de los apoyos que recibe de la escuela en la que trabaja. Diversos autores han destacado cómo el profesor principiante se enfrenta a la realidad laboral casi en una situación de shock, con dificultades que debe enfrentar y resolver con sus propios recursos y con los apoyos externos (Alliaud y Antelo, 2009; Alliaud 2004; Marcelo, 2008 y 2009; Veenman, 1984). Sin embargo, estas situaciones tan difíciles en un comienzo, cimentarán la base del camino que inevitablemente tienen que recorrer para convertirse en un profesor experto.

Otra idea que se releva en la investigación es que los profesores en este período son tanto aprendices como enseñantes; en la medida que desarrollan procesos de enseñanza ellos también aprender a enseñar (Feiman-Nemser, 2000). En este contexto, los profesores principiantes aprenden a conocer a sus estudiantes, a la institución, a las familias, el currículo escolar, además de asumir la responsabilidad del aprendizaje de sus estudiantes. En consecuencia, es un período que provoca tensiones, pero que también conlleva desafíos y aprendizajes, que favorece el desarrollo profesional y el equilibrio personal. En este período, el profesor principiante debe sopesar y balancear varias situaciones a la vez, de manera tal que en su conjunto contribuyan a su desarrollo profesional.

Asimismo, Marcelo (2009) hace referencia a una serie de investigaciones que han estudiado esta problemática. Por ejemplo, estudios en los que se incorpora la idea que empezar a trabajar en los establecimientos escolares es una experiencia dura, porque los principiantes se enfrentan a problemas tan variados como: la interiorización de las normas de una institución, el trabajo con pares y otros profesionales, la relación profesional con apoderados y el monitoreo de los comportamientos y del aprendizaje de los alumnos en el aula. Estos problemas, en ocasiones, estarían explicando la deserción o el abandono de la docencia en los primeros años (Veeman, 1984). En Estados Unidos, se han dado sugerencias con respecto a la creación de comunidades de aprendizaje en la escuela como una manera de enfrentar y contextualizar estas inevitables tensiones (Cochran-Smith, 2004). 
Respecto del tema, investigaciones de los años noventa (Huberman, Gonauer y Marti, 1993) ya revelaban que los principales problemas de los profesores novatos se categorizaban en problemas personales no vinculados a la enseñanza, de índole económico o familiar, y pedagógicos, por ejemplo, las relaciones con los alumnos, el dominio de los conocimientos que debe enseñar, las relaciones con sus colegas, las relaciones con la institución escolar, además de su preparación profesional.

En Uruguay, Rodríguez (2007) concluyó que la realidad de los profesores era similar a la que ocurre en otros países. Menciona que una dificultad relevante del primer año es la coacción de las autoridades de los establecimientos escolares por lograr determinados propósitos, lo que afecta la capacidad de los profesores novatos para tomar decisiones. También destaca que en muchas escuelas no existen pautas, orientaciones y estructuras para la coordinación de las actividades que hace el profesor. En muchos casos existiría un choque con la realidad escolar, porque no hay suficientes oportunidades en el aula para la innovación pedagógica lo que amilana al novato porque no tiene espacio para tomar decisiones autónomas.

En otra investigación acerca de los profesores principiantes en Estados Unidos (Thompson, 2007), se sistematizaron tres grupos de debilidades. El primero es el ámbito personal, cuyas dificultades estaban relacionadas con el manejo del tiempo, las interrelaciones personales, el asumir distintas labores en forma simultánea, el stress y nerviosismo frente a la observación, entre otros aspectos. El segundo es el ámbito de la planificación y manejo de la clase, en el cual las dificultades se relacionan con el desconocimiento de los lineamientos curriculares, apresuramiento por cubrir las materias escolares en vez de preocuparse del aprendizaje, insuficientes oportunidades de aprendizaje o la no consideración de los conocimientos previos y de los estilos de aprendizaje. El tercer ámbito está referido a la evaluación, las dificultades se resumen en escasez de evaluaciones formativas, diseño y construcción de instrumentos evaluativos adecuados para evaluar lo que efectivamente se enseñó y herramientas para evaluar el progreso de los estudiantes.

Otra problemática relacionada con profesores novatos en Canadá (St. Vicent y Gervais, 2010), está referida a los problemas éticos que deben afrontar en la escuela. Las investigadoras se preguntan si un profesor novel está preparado para afrontar problemas éticos en la escuela, concluyendo que cuando los profesores jóvenes analizan un problema educacional con dimensiones éticas, lo hacen contemplando consideraciones más bien relacionadas con la inserción profesional que con las dimensiones del problema ético propiamente tal.

Otros investigadores (Eirin et al., 2009), en un estudio exploratorio que indaga acerca de las dificultades de los profesores noveles, señalan que entre los retos inmediatos que deben encarar los profesores principiantes está la planificación, la organización y gestión del aula, el conocer a sus pares e implicarse en el centro, además de atender a la diversidad de alumnos y padres. Los autores clasifican las preocupaciones de los novatos en cuatro áreas: a) referidas a los alumnos, b) relacionadas con la docencia, c) relaciones con las familias y d) aspectos relacionados con el centro educativo.

En Chile, algunos investigadores realizaron una exhaustiva revisión bibliográfica relacionada con profesores principiantes, indicando que los problemas que viven los profesores al inicio de su vida profesional están referidos a la falta de tiempo para enseñar, el elevado número de alumnos por clase, la mala calidad de la infraestructura y la escasez de material de enseñanza (Ávalos, Carlson y Aylwin, 2004). En otro estudio, de carácter 
cualitativo, con 29 profesores principiantes chilenos, se señala que los desafíos centrales que ellos perciben son: dominar el conocimiento pedagógico del contenido, manejar la disciplina, considerar las necesidades individuales de los estudiantes, evaluar sus trabajos y manejar el currículo y recursos (Flores, 2014). Por su parte, otra investigación de carácter cuantitativo, con 890 profesores principiantes de enseñanza básica, reporta que la principal dificultad que perciben es el "Manejo de los estudiantes", concentrándose tal dificultad en los egresados de universidades altamente selectivas y en profesores que se inician en contextos vulnerables (Ruffinelli, 2013).

Estas dificultades y problemáticas abordadas por la investigación nacional e internacional han llevado a la creación, en numerosos países, de programas de inducción, de apoyo, de acompañamiento o de mentoría dirigidos a los profesores principiantes. Algunos son desarrollados e impulsados por el Estado y otros por las universidades, que progresivamente han ido comprendiendo que la responsabilidad formativa no concluye cuando egresa el profesor en formación.

Ávalos et al. (2004) confirman que los profesores principiantes en Chile tienen dificultades similares a los profesores noveles de otros países. Sin embargo, con respecto a otros países, la situación se agrava porque no es común que los chilenos reciban ayuda formal para abordar y dar solución a los problemas una vez que se insertan en los centros educativos. En general, los novatos adquieren experiencia y resuelven sus dificultades a través del ensayo y error, o solicitando apoyo informal a profesores más experimentados que quieren compartir su experiencia y apoyar a los más jóvenes.

Otro estudio relacionado con los profesores principiantes de Educación Básica en escuelas chilenas (Cisternas, 2011) establece que estas dificultades dependen de tres factores: a) las oportunidades que la formación inicial proporcionó para problematizar la realidad, b) las características de la inserción a la institución escolar y c) las condiciones personales del profesor principiante.

Resultados preliminares de una reciente investigación (Contreras, Vásquez, Rittershaussen, Solís y Núñez, 2013), con profesores novatos de enseñanza media egresados de dos universidades chilenas, en su primer año de experiencia laboral, permitieron detectar un conjunto de necesidades y dificultades que vivieron estos profesores al insertarse en los centro educativos. En términos generales, se establece que el factor tiempo es el problema más evidente: tienen mucho trabajo y poco tiempo para cumplir con todas las tareas demandadas; la multiplicidad de tareas los abruma y dicen sentirse poco preparados para asumirlas y hacerlas bien.

Por su parte, otra autora (Boerr, 2014) fundamenta la necesidad de programas de inducción para los profesores noveles en la complejidad de la tarea. Estos deben integrar los conocimientos teóricos a la práctica y responder a las demandas con soluciones rápidas, teniendo presente el conocimiento del contexto, la organización del tiempo, la conducción de los estudiantes con sus conflictos y el manejo de instrumentos curriculares.

Con respecto a investigaciones acerca de procesos de apoyo para profesores principiantes, en una investigación en Estados Unidos (Smith e Ingersoll, 2004) se señala que en los últimos años se produjo un aumento en el número de programas que ofrecían apoyo y orientación a los profesores en su primer año. El estudio analizaba si dichos programas tenían un efecto positivo sobre la retención de profesores principiantes en los establecimientos escolares. Los datos utilizados en el análisis indicaron que los principiantes que participaron en los programas, que tuvieron mentores en su disciplina y 
se integraron a las actividades colectivas de inducción, tales como planificación y trabajo colaborativo con otros profesores, eran más propensos a permanecer en la misma escuela y en la profesión.

Otro estudio relacionado con la efectividad de los programas de inducción y mentoría, a través de un método mixto, examinó los efectos de la asociación escuela-universidad en la inducción de profesores de primaria. Se investigaron los tipos de la ayuda proporcionados a los profesores principiantes y se determinó las tasas de retención después de un año en la profesión. Tanto los datos cuantitativos como cualitativos revelaron un aumento de las prácticas efectivas en el aula. Además, indicaron que los principiantes recibieron mayor apoyo de los mentores y todos ellos regresaron a la escuela en el segundo año (Davis y Higdona, 2008).

Por otra parte, en un estudio sobre procesos de mentoría se señala que esta es la instancia de vínculo entre la universidad y los centros educativos, y que debería ser parte formal de los planes de formación de profesores. Y agregan los autores: "la mentoría inserta en procesos de formación inicial docente se visualiza como una de las estrategias tendientes a generar espacios reales entre las instituciones formadoras, la escuela y el futuro profesor, es decir, conformar una tríada formativa, situando el aprendizaje docente en la acción" (Díaz y Bastías, 2013: 303).

En otro estudio acerca del acompañamiento a profesores noveles en su primer puesto de trabajo en Argentina, Alen plantea que "la función de acompañamiento reviste una alta productividad en lo que hace a las articulaciones entre la formación inicial y la formación continua. Pero, fundamentalmente articula de un modo innovador a las instituciones de formación docente con las escuelas de todos los niveles educativos de cada localidad" (2009: 81). El acompañamiento provee contextos grupales de análisis y reflexión del desempeño y sugieren la co-observación y los talleres de análisis y reflexión para los profesores noveles, además de estrategias de articulación para programas de mentoría y formación de mentores, entre ellas seminarios para estudiar y explorar acciones de acompañamiento, la publicación de experiencias para sistematizar el corpus conceptual y práctico y un aula virtual de acompañamiento con un banco de recursos para los formadores.

Otros autores (Ingersoll y Strong, 2011) publicaron una revisión de 15 estudios, realizados desde mediados 1980 en adelante, sobre los efectos del apoyo, guía y orientación de los programas de inducción para profesores principiantes en Estados Unidos. La mayoría de ellos proporcionan información detallada que confirman la tesis de que el apoyo y la asistencia de los profesores principiantes tiene un impacto positivo en tres ámbitos: compromiso y retención de los profesores en la profesión, mejor práctica de enseñanza y mayores logros de aprendizaje de los estudiantes. Sobre el compromiso y la retención, se comprobó que la mayor parte de los profesores principiantes que participaron en los programas de inducción permanecieron en la profesión. En cuanto a mejores prácticas de enseñanza, la mayoría de los estudios revisados mostraron que los profesores principiantes realizaron mejores clases, mantuvieron la atención de los estudiantes en las tareas, incentivaron la reflexión de los estudiante, hicieron ajustes a las actividades de las clases para satisfacer mejor los intereses de los estudiantes, mantuvieron un ambiente de aula positivo y fueron capaces de manejar exitosamente el aula. Finalmente, casi todos los estudios mostraron que los estudiantes de dichos profesores alcanzaron resultados de aprendizaje más altos en las pruebas de rendimiento académico. 
A modo de síntesis, es posible plantear que tanto las investigaciones nacionales como internacionales realizadas en los últimos treinta años coinciden en lo siguiente: a) la inserción profesional docente es un período muy difícil de abordar debido a la multiplicidad de problemáticas que afectan a los docentes principiantes; b) los problemas que afectan a los profesores jóvenes al inicio de la vida laboral son coincidentes en diferentes investigaciones; c) los problemas que afectan a los profesores principiantes derivan de la formación inicial y también de las condiciones de cada centro educativo; d) hay consenso en la necesidad de apoyar la inserción de los profesores novatos con programas de acompañamiento que deberían abarcar el período de iniciación a la enseñanza, es decir aquel en que se produce el tránsito entre estudiante y profesor.

\section{METODOLOGÍA}

Los resultados presentados en este artículo son parte de una investigación más amplia (Fondecyt 1131091) cuyo diseño consideró un estudio transeccional y otro longitudinal. Para el primero, la muestra estuvo constituida por 198 profesores novatos, de los cuales 96 son profesores de educación básica $(48,5 \%)$ y $102(51,5 \%)$ de educación media, egresados de 6 instituciones formadoras de profesores en Chile y que se desempeñaban en establecimientos educacionales de diferente dependencia, ubicados en cuatro regiones del país.

La muestra es voluntaria y se contó con bases de datos de los egresados de cada universidad en los tres últimos años. Estos profesores fueron invitados a participar en la investigación, siendo requisito fundamental el que estuviesen trabajando en un establecimiento educacional. Los profesores novatos que respondieron afirmativamente fueron contactados para darles a conocer los alcances de la investigación y finalmente acordar la formalización de su participación.

Para el segundo estudio, de carácter longitudinal, se contó con una muestra voluntaria de 20 profesores novatos que ya habían participado en el estudio transeccional, a los cuales se les observó clases para analizar su desempeño y, posterior a cada una de ellas, se les realizó una entrevista para indagar acerca de las problemáticas vivenciadas en su trabajo y que afectan su desempeño.

El análisis de datos, cuyos resultados se presentan en este artículo, fue tanto de tipo cuantitativo como cualitativo, dependiendo del tipo de información recogida.

El primer análisis, de carácter cuantitativo, se realizó a partir de la información proveniente de un cuestionario que permitió conocer los problemas que afectan el desempeño de los profesores principiantes en sus primeros años de ejercicio docente, los cuales participaron del estudio transeccional. Este cuestionario contenía tres apartados: Desempeños profesionales, Condiciones de trabajo para el desempeño profesional, Apoyos para la inserción profesional.

En este artículo se da cuenta de la información recabada en el apartado de Desempeños profesionales, constituido por 19 afirmaciones que respondían a diferentes desempeños propios del quehacer docente, las cuales se presentan en la Tabla 1. 
Tabla 1. Tareas o desempeños profesionales.

\begin{tabular}{|ll|}
\hline 1. & Toma de decisiones curriculares y didácticas respecto a la planificación de la enseñanza. \\
\hline 2. & Toma de decisiones respecto de la planificación de la evaluación. \\
\hline 3. & $\begin{array}{l}\text { Relación entre la conducción del proceso enseñanza y la generación de clima facilitador del } \\
\text { aprendizaje. }\end{array}$ \\
\hline 4. & Conducción de actividades a distintos niveles de complejidad. \\
\hline 5. & Conducción de actividades variadas. \\
\hline 6. & Uso de recursos variados. \\
\hline 7. & Manejo de estrategias para captar la atención de los alumnos. \\
\hline 8. & Manejo de estrategias para involucrar a los alumnos en el aprendizaje. \\
\hline 9. & Tratamiento de los contenidos. \\
\hline 10. & Uso de instrumentos de evaluación. \\
\hline 11. & Uso de recursos didácticos diferenciados. \\
\hline 12. & Uso de recursos evaluativos diferenciados. \\
\hline 13. & Trabajo con los apoderados. \\
\hline 14. & Interacción profesional con colegas y directivos. \\
\hline 15. & Manejo de estrategias para insertarse como profesor en la institución. \\
\hline 16. & Ejecución de tareas de manera simultánea en el aula (clases, revisión de cuadernos, completar \\
& formularios y libro de clases, etc.). \\
\hline 17. & Ejecución de tareas variadas en la institución (talleres, actos, atención apoderados, clases, \\
& reuniones, etc.). \\
\hline 18. & Manejo de problemáticas sociales contingentes. \\
\hline 19. & Manejo de situaciones de alto riesgo. \\
\hline
\end{tabular}

Se utilizó una escala de respuesta con cuatro categorías asociadas al nivel de coherencia de la preparación entregada por la institución formadora para el desempeño de esa tarea. Se consideran como problemas aquellos desempeños profesionales en que más del $50 \%$ del total de profesores novatos, dicen no sentirse preparados para su ejecución.

Para complementar la tarea relativa a "Ejecución de tareas variadas en la institución", se analizaron las respuestas dadas por los profesores novatos a una pregunta abierta del cuestionario, en la que se les solicitaba especificar qué tareas extra aula realizaban en la institución. A partir de las respuestas de levantaron siete categorías que corresponden a: Tareas asociadas a jefatura de curso, Tareas pedagógicas con pares, Talleres con alumnos, Tareas de preparación del trabajo en aula, Tareas administrativas de curso, Turnos y reemplazos y, finalmente, Tareas de gestión.

El instrumento fue aplicado a una muestra voluntaria de 198 profesores novatos, de los cuales 96 son profesores de educación básica (48,5\%) y 102 (51,5\%) de educación media. Del total de profesores novatos, el 48,5\% estaban en su primer año de inserción, el 22,7\% en segundo año y el $28,8 \%$ en tercer año de trabajo.

Para el segundo análisis, de carácter cualitativo, se consideraron las respuestas dadas por los profesores novatos, participantes del estudio longitudinal, a la pregunta ¿Cuáles son los problemas que han afectado tu desempeño durante este período de trabajo? Esta pregunta formó parte del conjunto de interrogantes planteadas en la entrevista realizada por 
los investigadores a los profesores novatos, una en cada año que duró el estudio.

Las respuestas permitieron confirmar la información obtenida del cuestionario respecto a los problemas percibidos por los profesores en diferentes dimensiones de su quehacer y detectar si estos problemas persisten a lo largo del tiempo o surgen otros.

\section{RESULTADOS}

Los resultados de este estudio dan cuenta de la percepción que tienen los profesores principiantes con respecto a los problemas que afectan su quehacer docente en las instituciones educativas en que se encuentran insertos. Los datos analizados fueron recabados a través de un cuestionario para el grupo que participó en el estudio transeccional y de una entrevista para los que participaron del estudio longitudinal.

En el cuestionario se entregaron 19 premisas que correspondían a diversos desempeños o tareas relacionadas con la vida laboral. Los profesores debían indicar el grado de coherencia entre la formación dada en la universidad y lo demandado por la institución educativa. Fueron considerados problemas aquellas tareas o desempeños en los que más del 50\% de profesores manifestó baja coherencia.

En el Gráfico 1 se pueden apreciar aquellas tareas en que más de la mitad de los profesores novatos perciben no estar preparados: uso de recursos didácticos diferenciados $(52,5 \%)$, uso recursos evaluativos diferenciados $(57,6 \%)$, trabajo con los apoderados $(75,8 \%)$, ejecución de tareas de manera simultánea en aula $(51,0 \%)$, ejecución de tareas variadas en la institución (60,1\%), manejo de problemáticas sociales contingentes $(54,5 \%)$, manejo de situaciones de alto riesgo $(68,2 \%)$ y bordeando el $50 \%$ manejo de estrategias para insertarse como profesor en la institución (49,5\%).

Gráfico 1. Tareas problemas de profesores novatos

\section{Tareas Problema de Profesores Novatos}

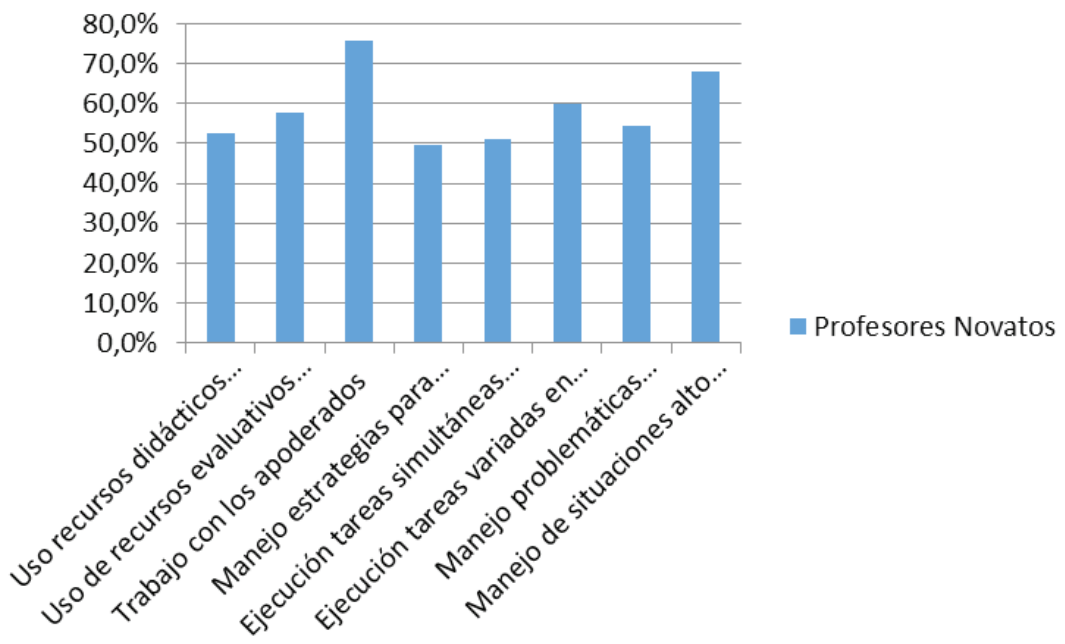


Al analizar las tareas o desempeños percibidos por los profesores novatos como más deficientes, en cada año de inserción profesional (Tabla 2), llama la atención que en algunas tareas se mantiene el alto porcentaje de profesores novatos que perciben no estar preparados adecuadamente para desarrollarlas, esto es independiente de los años de ejercicio profesional, incluso en algunas de ellas el número de profesores principiantes aumenta en el tercer año. Por ejemplo, el trabajo con apoderados, la ejecución de tareas variadas en la institución y el manejo de situaciones variadas de alto riesgo. Lo anterior indicaría que la inserción en la escuela tampoco los ayuda a enfrentar mejor esas tareas; en consecuencia el profesor novato carecería de herramientas para afrontar dichos desempeños.

Tabla 2. Tareas o desempeños problema según año de inserción

\begin{tabular}{|l|l|l|l|}
\hline Tareas problema & Año 1 & Año 2 & Año 3 \\
\hline Uso de recursos didácticos diferenciados & $55,2 \%$ & $46,7 \%$ & $52,6 \%$ \\
\hline Uso de recursos evaluativos diferenciados & $55,2 \%$ & $60,0 \%$ & $59,6 \%$ \\
\hline Trabajo con apoderados & $79,2 \%$ & $68,9 \%$ & $75,4 \%$ \\
\hline Manejo de estrategias para insertarse en la institución & $49,0 \%$ & $44,4 \%$ & $54,4 \%$ \\
\hline Ejecución de tareas simultáneas en aula & $52,1 \%$ & $44,4 \%$ & $54,4 \%$ \\
\hline Ejecución de tareas variadas en la institución & $61,5 \%$ & $44,4 \%$ & $72,2 \%$ \\
\hline Manejo de problemáticas sociales contingentes & $53,0 \%$ & $55,6 \%$ & $56,1 \%$ \\
\hline Manejo de situaciones alto riesgo & $62,5 \%$ & $71,1 \%$ & $75,4 \%$ \\
\hline
\end{tabular}

Con respecto a lo relacionado a la tarea "Ejecución de tareas variadas en la institución" (Tabla 2), la información se complementa con la recabada en una pregunta abierta del cuestionario, en la cual se solicitaba al profesor novato que especificara las tareas extra aula que debían realizar en el establecimiento. Al revisar las respuestas dadas por los novatos, se observa que mencionan diversas tareas que deben abordar más allá de la docencia en aula; algunas tareas mencionadas ejemplifican la diversidad de actividades que realizan en el establecimiento educacional:

Planificar, preparar materiales, revisar cuadernos y evaluaciones. Consejo de profesores y actividades a nivel colegio, gestión de actividades con comunidad, salidas educativas y confeccionar informes de notas, redactar informes de personalidad (Caso 109).

Turnos de observación en el almuerzo, corrección de evaluaciones y planificación de clases y actividades (Caso 118).

Reuniones de apoderados, actividades extra programáticas generadas para producir fondos u otras situaciones para el curso de la jefatura, consejo de profesores de educación media y consejo general del colegio. Entrevistas continuas con los apoderados y trámites administrativos (Caso 171). 
Reuniones de apoderados, turnos de patio, olimpiadas padre-hijo, graduación 8vo-IV medio, preparar escenografía día del libro, preparar bailes para actos, olimpiadas de profesores, primera comunión, misas, fiesta de la chilenidad y premiaciones por nivel (Caso 201).

Asistir a eventos como pentecostés, misas, ceremonias de graduación, día de la chilenidad, olimpiadas, organización día del colegio, toma de pruebas atrasadas fuera del horario de clases, pintar escenografía o práctica de coreografía propias de profesores, análisis de evaluaciones, revisar informes, entrevistas de apoderados y alumnos (Caso 210).

Para profundizar en el análisis de las tareas extra aula antes mencionadas, las respuestas de los profesores se organizaron en ocho categorías, las que se presentan en la siguiente tabla:

Tabla 3. Categorías de tareas extra aula

\begin{tabular}{|c|c|}
\hline Categorías de tareas extra aula & Tareas extra aula \\
\hline 1 Tareas asociadas a jefatura de curso & $\begin{array}{l}\text { Atención a estudiantes, Tutoría alumnos, Reuniones y } \\
\text { atención de apoderados. }\end{array}$ \\
\hline 2 Tareas pedagógicas con pares & $\begin{array}{l}\text { Reuniones con: orientador o psicólogo, UTP, } \\
\text { profesores del ciclo, todos los profesores, con } \\
\text { profesores nuevos (tutoría). }\end{array}$ \\
\hline 3 Talleres con alumnos & $\begin{array}{l}\text { Talleres extra programáticos, salidas culturales, de } \\
\text { reforzamiento, vocacionales, programas de integración, } \\
\text { talleres TIC, taller SIMCE. }\end{array}$ \\
\hline 4 Tareas de preparación del trabajo en aula & $\begin{array}{l}\text { Planificación de clases, diseño de materiales, } \\
\text { preparación de evaluaciones, corrección de } \\
\text { evaluaciones, evaluación dominio lector. }\end{array}$ \\
\hline 5 Tareas administrativas de curso & $\begin{array}{l}\text { Elaboración de informes, libretas de notas, asistencia, } \\
\text { revisión de comunicaciones, tomar pruebas atrasadas. }\end{array}$ \\
\hline 6 Turnos y reemplazos & $\begin{array}{l}\text { Turnos de patio, de almuerzo, en furgón escolar, } \\
\text { reemplazo de colegas }\end{array}$ \\
\hline 7 Tareas de gestión & $\begin{array}{l}\text { Encargado de biblioteca, asesor centro de alumnos y } \\
\text { de centro de padres, participación en comité } \\
\text { aniversario, comité bipartito, apoyo proyecto de } \\
\text { integración. }\end{array}$ \\
\hline $\begin{array}{l}8 \text { Tareas de preparación y participación en } \\
\text { eventos propios del establecimiento }\end{array}$ & $\begin{array}{l}\text { Participación en misas, retiros espirituales, actos } \\
\text { cívicos, celebraciones del colegio, completación de } \\
\text { horas el día sábado. }\end{array}$ \\
\hline
\end{tabular}


Considerando la muestra total de profesores novatos del estudio transeccional, se obtiene que las categorías de tareas extra aula (Gráfico $\mathrm{N}^{\circ} 2$ ) más mencionadas por los profesores novatos $(34,5 \%)$ están relacionadas con las demandas de la jefatura de curso (categoría de tarea 1) como, por ejemplo, atender a alumnos y apoderados, realizar tutorías y reuniones de apoderados, y las asociadas a preparación o participación en eventos distintivos de cada centro educativo (categoría de tarea 8): actos litúrgicos, celebraciones varias, retiros espirituales, entre otros.

En tanto, las tareas menos mencionadas $(10,6 \%)$ son las administrativas de curso (categoría de tarea 5). Por ejemplo, realizar informes, tomar pruebas atrasadas o mantener al día el libro de clases. Las categorías de tareas restantes se distribuyen de la siguiente manera: 25,8\% nombra las tareas asociadas a gestión (categoría de tarea 7), el 16\% indica realizar turnos o reemplazos (categoría de tarea 6), el 27\% señala especifica que realiza tareas de planificación, diseño y selección de materiales (categoría de tarea 4), en tanto que el $21 . \%$ se refiere a reuniones con pares (categoría de tarea 2) y el 18,2\% dice participar en la realización de talleres para los estudiantes (categoría de tarea 3).

Gráfico 2. Categorías de tareas extra aula abordadas por el total de profesores novatos

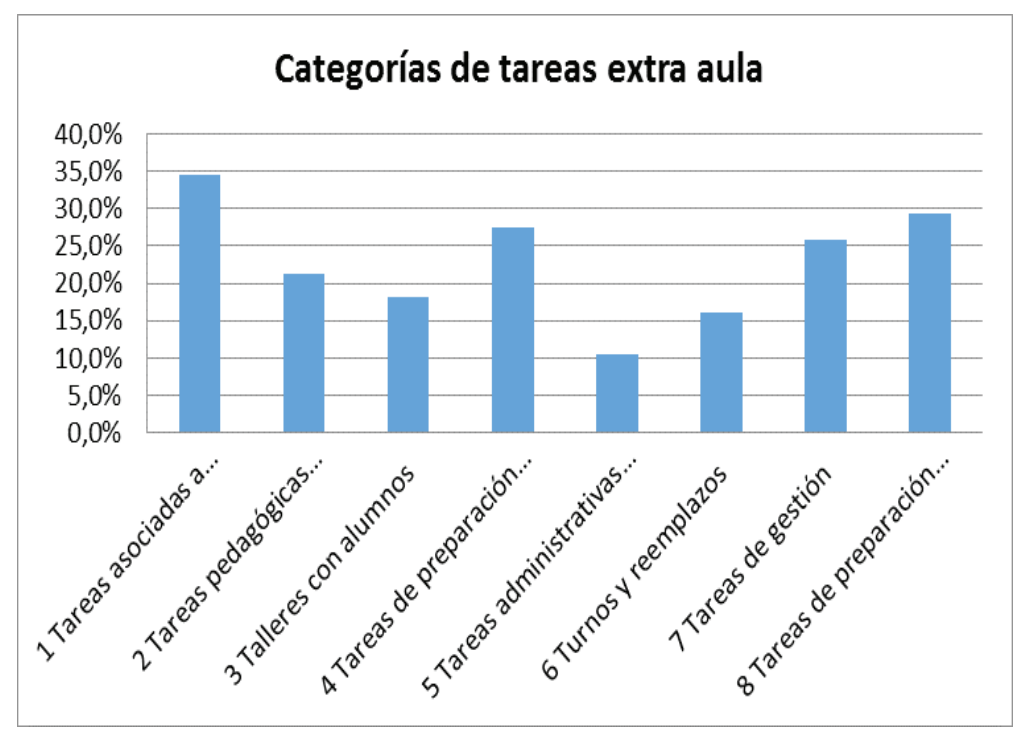

Al realizar el análisis de las tareas, según el título del profesor novato (Educación Básica o Educación Media), se aprecia que varía el tipo de categoría de tareas extra aula (Gráfico 3). Los profesores novatos de básica reportan participar más en tareas asociadas a la jefatura de curso (categoría de tarea 1) y a eventos propios del centro (categoría de tarea 8), en tanto que los profesores de media reportan más tareas asociadas a la preparación de la enseñanza (categoría de tarea 4). También varía la realización de turnos y reemplazos (categoría de tarea 6) que es informada por el $9 \%$ de profesores de Media y por el $22 \%$ de profesores de Básica. 
Gráfico 3. Categorías de tareas extra aula realizadas por profesores novatos de educación básica y de educación media.

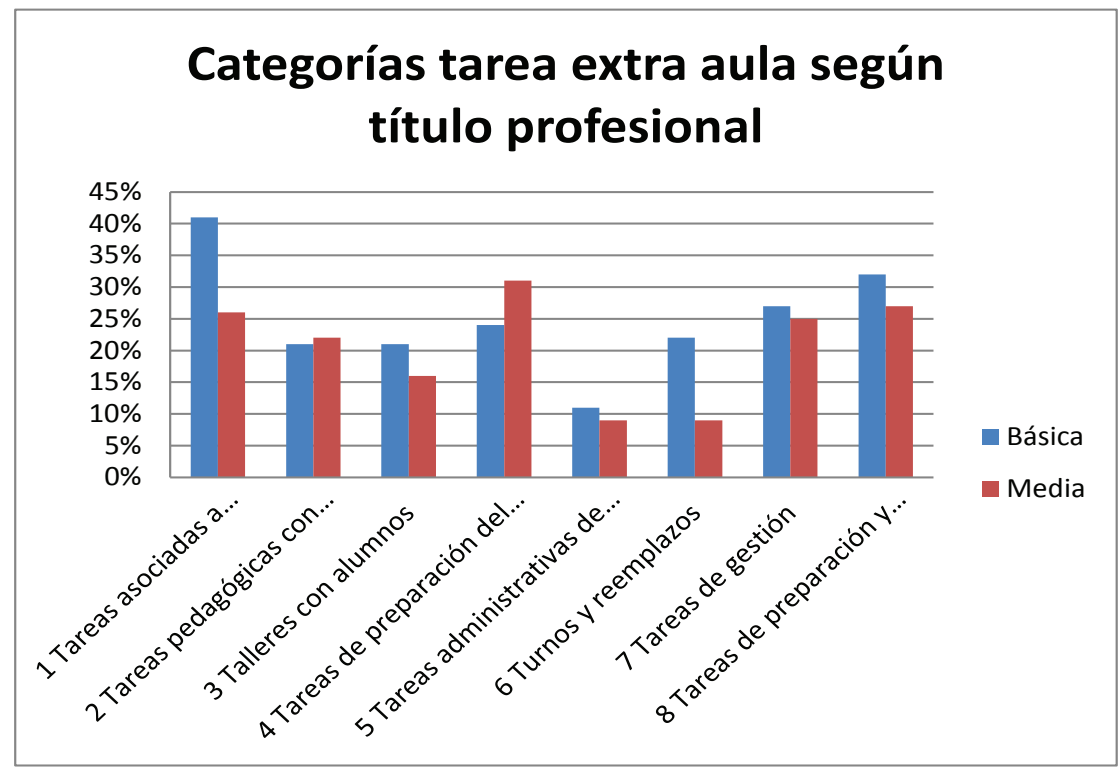

De los análisis anteriores se percibe que, sin lugar a dudas, los profesores novatos deben enfrentar la problemática de realizar una multiplicidad de tareas que van más allá de la realización de clases. Las tareas relacionadas con las categorías 1, 4, 7 y 8 son las que afectan a más profesores novatos, es decir, aquellas asociadas a la jefatura de curso, preparación del trabajo en aula, tareas de gestión y a la de preparación y participación en eventos propios del establecimiento.

A partir de las entrevistas realizadas a los participantes del estudio longitudinal, a los cuales se les realizó un seguimiento durante tres años y en cada uno de ellos se les entrevistó con el fin de conocer sus problemas en el proceso de inserción profesional y en su desempeño docente, así como en la forma de solución de los mismos, se observan los siguientes resultados.

Los problemas mencionados por los profesores novatos durante el primer año de trabajo están relacionados con las dificultades que ellos tienen para manejar y conducir al grupo de alumnos, el trabajo con apoderados, los problemas administrativos por el desconocimiento de la multiplicidad de tareas que deben realizar en la escuela más allá de la docencia en aula, la dificultad que les significa seleccionar contenidos para la planificación, lograr los estándares de aprendizaje determinados por pruebas estandarizadas y la selección de estrategias adecuadas para trabajar con alumnos con necesidades educativas especiales.

Al término de su segundo año, algunas problemáticas se repiten, por ejemplo la dificultad para manejar grupos, el trabajo con alumnos con necesidades educativas especiales, la multiplicidad de tareas que realizan más allá de la docencia en aula. Sin embargo aparecen 
otras preocupaciones tales como la toma de conciencia de que los tiempos reales de enseñanza son mínimos, debido a la diversidad de tareas que el profesor realiza en aula, por ejemplo pasar la lista, revisar tareas y comunicaciones, entre otros. También surge un caso en que la profesora no se siente bien preparada en una de las asignaturas que imparte y eso la ha llevado a tener que buscar apoyo en profesores de otros establecimientos, los que a su vez fueron sus profesores en el colegio.

En la entrevista realizada al tercer año, llama la atención que los problemas mencionados con anterioridad se repiten. Por ejemplo, el trabajo con padres, el trabajo con alumnos con necesidades educativas especiales, el tiempo real de enseñanza por la diversidad de tareas en aula que aborda el profesor, la excesiva carga de trabajo intra y extra aula que, según informa la mayoría de los casos, los obliga a continuar trabajando en casa y aun así no logran tener todo al día, como por ejemplo la planificación diaria. El manejo de grupo aparece como un problema, pero con menor frecuencia y los profesores que ya no lo mencionan dicen que el sentirse más empoderados como profesores los ha llevado a un mejor manejo y conducción de la clase.

También surgen dos casos que señalan como problemática para su desempeño la falta de preparación en áreas específicas para las cuales los profesores no recibieron formación en la universidad, de acuerdo con los requerimientos del lugar de trabajo; en ambos casos son profesores de educación básica. Uno de los casos realiza clases de música y el otro se desempeña en educación técnico profesional. Ambos profesores han buscado ayuda en colegas externos al establecimiento y han estudiado por su propia cuenta, sin el apoyo del establecimiento educativo en el que trabajan.

Algunos comentarios de los profesores novatos que dan cuenta de las problemáticas vividas en los tres primeros años se incluyen en la siguiente tabla.

Tabla 4. Tareas problema señalados por los profesores novatos

\begin{tabular}{|l|l|}
\hline Tareas problema & Comentarios planteados por profesores \\
\hline Trabajo con padres & $\begin{array}{l}\text { La mayor dificultad es el trato con apoderados, en la universidad no se nos } \\
\text { enseñó cómo trabajar con ellos y los apoderados de este colegio son } \\
\text { difíciles, hasta insolentes con el profesor. Algunos me decían que como era } \\
\text { nueva no sabía hacer las cosas; los papás eran atrevidos y se aprovechaban } \\
\text { de mi inexperiencia, el colegio da mucha entrada a los papás, algunos } \\
\text { llegaban de mal modo, tres veces en el año quedé llorando luego de } \\
\text { conversar con padres (Caso 201). }\end{array}$ \\
\hline $\begin{array}{l}\text { Problemas adminis- } \\
\text { trativos y multiplici- } \\
\text { dad de tareas extra } \\
\text { aula }\end{array}$ & $\begin{array}{l}\text { Principalmente problemas administrativos, más que problemas son nada } \\
\text { tareas que tienes que asumir en la práctica y que ni se mencionaron en la } \\
\text { universidad. Nosotros sabíamos que íbamos a tener que planificar clase a } \\
\text { clase, que teníamos que atender apoderados, las reuniones, sabía que el } \\
\text { libro tenía una forma de llenarse, pero no sabía que el llenado del libro } \\
\text { tenia tantos detalles como la asistencia, los certificados, dejar anotado al } \\
\text { reverso del libro, además semana a semana nos vamos rotando para llevar } \\
\text { el avance curricular del colegio, también con el registro de las actividades } \\
\text { que se van haciendo (Caso 221). }\end{array}$ \\
\hline
\end{tabular}




\begin{tabular}{|c|c|}
\hline $\begin{array}{l}\text { Tiempo real de en- } \\
\text { señanza y multipli- } \\
\text { cidad de tareas intra } \\
\text { aula }\end{array}$ & $\begin{array}{l}\text { Clases muy difíciles de desarrollar en mi caso por ejemplo el problema es } \\
\text { cuan efectivo es el tiempo de una clase de } 45 \text { minutos, cuánto es lo que tú } \\
\text { alcanzas a desarrollar y esos son } 25 \text { minutos de clase. Los otros } 20 \text { minutos } \\
\text { se pierden en que viene el auxiliar a entregarte el libro, después viene el } \\
\text { mismo auxiliar a retirar el libro, en que vienen a tal o cual cosa a } \\
\text { interrumpir la clase y eso es de los actores periféricos. Ahora también se } \\
\text { pierde en lograr en el aula las condiciones necesarias para desarrollar tu } \\
\text { clase (Caso 604). }\end{array}$ \\
\hline $\begin{array}{l}\text { Trabajo con alum- } \\
\text { nos diversos }\end{array}$ & $\begin{array}{l}\text { En la universidad solo abordan aprendizajes para todos y no me enseñaron } \\
\text { estrategias para distintos niveles; tampoco me enseñaron a trabajar } \\
\text { problemas de conducta y en la práctica estuve en colegios con niños } \\
\text { tranquilos, con buenos comportamientos. En el colegio hay Proyecto de } \\
\text { integración (PEI); en la U como estábamos juntas con estudiantes de } \\
\text { diferencial sínos habían dicho lo que era un PEI (Caso 604). } \\
\text { De las dificultades nuevas o que han ido aumento de las que mencioné el } \\
\text { año pasado es la que se refiere a trabajar con niños con problemas de } \\
\text { aprendizaje, nosotros no estamos preparados para trabajarcon Necesidades } \\
\text { de aprendizajes. Este año, mejor dicho el año pasado empezó a trabajar } \\
\text { con un proyecto de integración PIE. El año pasado teníamos tres o cuatro } \\
\text { alumnos por sala que estaban diagnosticados o que tenían problemas de } \\
\text { aprendizaje, pero hoy tenemos más niños con problemas y algunos } \\
\text { encubiertos, con necesidades de aprendizaje y eso dificulta nuestro trabajo. } \\
\text { Yo como profesional de la educación y en mi caso de matemática estoy } \\
\text { preparada para trabajar con un nivel estándar o promedio, no estoy } \\
\text { preparada para trabajar con niños que tienen autismo... y eso ha sido un } \\
\text { proceso largo y demoroso. Ahora estamos en un proceso de conocerlos, } \\
\text { conocer sus características y como ayudarlos y mejorar su aprendizaje y } \\
\text { como abordar todas las áreas en la sala de clases y poder decir que esta } \\
\text { actividades visual, kinésica y que las tenga todas para ayudar y llamar la } \\
\text { atención de todos (Caso 407). }\end{array}$ \\
\hline Manejo de grupo & $\begin{array}{l}\text { Lo que yo sé que me cuesta, es el manejo de grupo, y lo he recalcado en } \\
\text { todas las entrevistas, porque como lo dije en la entrevista anterior, en mi } \\
\text { universidad no nos enseñaron estrategias de grupo. Entonces cuando uno } \\
\text { va a observar clases, hace su práctica profesional, siempre está el profesor } \\
\text { jefe en el aula. Entonces uno no se desenvuelve por si sola dentro del aula. } \\
\text { Entonces si hay un profesor jefe, los niños se mantienen más controlados } \\
\text { que si no estuviese, y yo estuviese sola con el curso (Caso 405). } \\
\text { Las nuevas dificultades tuvieron relación con la conducta de los niños. Me } \\
\text { tocó una jefatura donde los niños eran irrespetuosos y bastante } \\
\text { garabateros. Además de esto, eran muy conversadores y contestatarios, lo } \\
\text { que dificultaba la realización de la clase. Los profesores de otras } \\
\text { asignaturas me reclamaban mucho por el curso, lo que muchas veces } \\
\text { generaba frustración, pues conmigo se portaban bien y con el resto de los } \\
\text { profesores mal (Caso 201). }\end{array}$ \\
\hline
\end{tabular}


Al finalizar la tercera entrevista a cada uno de los profesores novatos se les mencionó un listado de problemas que se levantaron de las respuestas dadas en las primeras entrevistas y se les preguntó cuál o cuáles de los problemas aun persistían.

El problema que más se repite está relacionado con el trabajo con alumnos diversos y con necesidades educativas especiales, como se aprecia en los siguientes comentarios:

En general hay dificultad en bajar el nivel de contenidos al grupo de alumnos. El trabajo con estudiantes diversos es algo que falta (Caso 255).

Trabajo con estudiantes diversos, esto está pendiente, traté de trabajar con inteligencias múltiples...debo adaptar los instrumentos para los niños de este nivel.

Sería interesante saber cómo trabajar con estudiantes diversos, quizás con otras creencias religiosas, o con distinta orientación sexual o que vengan de familias un poco más libres (Caso 461).

Persiste el problema del aprendizaje con estudiantes diversos, por ejemplo Joaquín el estudiante con Asperger ya estaba aburrido y preguntaba la hora de salida... hay que conversarle, mirarlo a los ojos, decirle que en x tiempo. Porque si se le dice 5 minutos tienen que ser 5 minutos... todo esto debo consultarlo porque no lo sé (Caso 604).

Tengo problemas con algunos alumnos hiperactivos, con alumnos diversos me faltan estrategias (Caso 650).

Otro de los problemas mencionados es el manejo de situaciones de riesgo y problemáticas sociales contingentes:

En el caso de las situaciones de riesgo, me tocó una vez que una niña llegó con droga al colegio y en ese momento no sabía qué hacer más que derivarlo a dirección. No me siento preparada para contener a los niños en esos momentos o cuando sufren violencia física y/o psicológica (Caso 461).

Lo de situaciones complejas es difícil, por ejemplo lo del bullying, como hacer entender a un papá que su hijo es agresor; es complejo (Caso 405).

Hoy se ve mayor nivel de agresividad, han llegado niños con pistolas, otro se arrancó, parecen cosas aisladas pero son repetitivas. No hay protocolos establecidos y para una cosa se hace algo y para otra se hace otra cosa. Al final uno no sabe cómo actuar (Caso 405).

Algo que también estoy trabajando este año, de manera voluntaria, es con alumnos desertores, entonces estoy viendo porque no quieren ir al colegio (Caso 461).

Otra dificultad es la referida al trabajo con padres y apoderados, pero específicamente en situaciones que afectan a alumnos en particular:

Los padres nunca quieren ver lo negativo de su hijo y si uno se los dice ellos se sienten mal y dicen que el colegio es el culpable porque eso no sucede en la casa y que todos 
son angelitos... cuesta atacar ahí y lograr un cambio porque la familia no apoya (Caso 405).

Otra dificultad fue lidiar con papás muy complicados en algunos casos (esto se reflejaba en sus hijos). Eran difíciles porque sus hijos no eran aceptados en el curso, ya que eran niños que se portaban muy mal y existía cierto rechazo hacia ellos. Los papás eran violentos al tratar conmigo (Caso 201).

Finalmente, algunos de los profesores novatos entrevistados dan algunas sugerencias a sus instituciones formadoras, con el fin de cautelar una mejor preparación y enfrentar los problemas surgidos en los tres primeros años de ejercicio profesional:

Recomendaría hacer un estudio sistemático de la malla y de lo que se quiere lograr en el perfil, pero faltan cosas más contextuales y no sólo ver la evolución de la didáctica, por ejemplo inteligencias múltiples, como lidiar con distintos niños, cómo trabajar con el tema de diversidad. Y generar un trabajo más cercano con los centros de práctica para conocer la realidad, que las clases son de 45 minutos en música, que se debe hacer clases en varios cursos, que sea todo más funcional así uno lo vería como más útil. También sería interesante saber lo que ocurre en las escuelas, por ejemplo Ley SEP, planes de los establecimientos; entonces dar herramientas a los estudiantes para que sean agentes de cambio (Caso 221).

Que tengamos más instancias de práctica, por ejemplo un semestre más de práctica. Para apoyar la formación en cuanto a manejo de aula. También apoyo para trabajar con niños con necesidades especiales, apoyo para trabajar con la diversidad de aula y estrategias para realizar jefatura de curso y manejo administrativo (Caso 255).

Que nos hubiesen hecho un ramo mínimo que nos dijera con qué tipo de niños nos podemos encontrar en la sala... actualmente son diversos. En la universidad te dicen este curso, pero no te dicen que puede haber un asperger o uno con autismo o limítrofe (Caso 604).

Quizás hubiese sido bueno tener más herramientas para saber cómo modificar una evaluación o qué intencionar en cada niño dependiendo de lo que necesito (Caso 201).

Deberían abrirse a que tengamos psicología I y II sino que también tengamos estrategias para trabajar con alumnos con necesidades diversas. Falta dar orientaciones a los profesores de cómo abordar el trabajo con alumnos con diferentes problemas de aprendizaje o que son más aventajados. No basta solo que se tengan cursos de psicopedagogía sino que también en la didáctica se den metodologías de cómo enseñar los temas de la asignatura (Caso 650). 


\section{CONCLUSIONES Y PROYECCIONES}

Los problemas reportados por los profesores novatos del estudio transeccional, por medio de un cuestionario, no difieren de aquellos identificados por los novatos del estudio longitudinal obtenidos de una entrevista y tampoco cambian a lo largo de los tres años que se realizó el seguimiento a estos últimos; es más, los problemas que persisten al tercer año ya fueron reconocidos al primer año de ejercicio profesional.

En este estudio se vislumbra que los problemas en la inserción profesional de profesores corresponden a dos áreas. La primera área de problemas que afecta al desempeño de los profesores novatos está relacionada con la formación inicial de profesores entregada por las universidades y puede ser analizada desde el Marco de la Buena Enseñanza, MBE (Mineduc, 2008), documento chileno que establece lo que los docentes del país deben conocer, saber hacer y ponderar, para determinar cuán bien lo hace cada uno en el aula y en la escuela. Este documento presenta 20 criterios que caracterizan un buen desempeño de los docentes, a partir de la experiencia práctica y el conocimiento científico, los cuales se agrupan en cuatro dominios con sus correspondientes descriptores. Los problemas que reportan los profesores novatos están referidos a las cuatro dimensiones del MBE.

Con respecto al dominio B, Creación de un ambiente propicio para el aprendizaje, referido "al ambiente y clima que genera el docente, en el cual tienen lugar los procesos de enseñanza y aprendizaje" (Mineduc, 2008: 9), los novatos en sus primeros años de inserción manifiestan tener dificultades para manejar al grupo de alumnos al momento de enseñar. Dicen que no conocen estrategias para centrar la atención, además de tener dificultades para mantener normas y crear un espacio que facilite el aprendizaje.

En cuanto al dominio C, Enseñanza para el aprendizaje de todos los estudiantes, referido a la generación de oportunidades de aprendizaje para todos los estudiantes, y la faceta A, Preparación para la enseñanza, los profesores novatos señalan que uno de los problemas es lograr que todos los estudiantes aprendan. Manifiestan falta de preparación para seleccionar recursos evaluativos y de enseñanza que respondan a la diversidad de alumnos, que no conocen las diferentes maneras de aprender de los estudiantes y que no están preparados para trabajar con estudiantes diversos, tanto en términos de diferencias culturales como de aprendizajes y, específicamente, que no se les preparó para enseñar a niños con necesidades educativas especiales.

Finalmente, en la faceta D, Responsabilidades profesionales, una de las debilidades es el trabajo con padres y apoderados. Ellos señalan que en la formación de profesores hay escasas instancias de formación en esta área y que mientras realizaron su práctica tampoco tuvieron oportunidades que les permitieran interactuar con los padres, por ejemplo, realizar entrevistas, participar en reuniones con apoderados o realizar otras tareas que propicien un trabajo más colaborativo con la familia.

En suma, los problemas manifestados por los profesores novatos están relacionados con todas las facetas del MBE. Si se toma conciencia que este documento no solo explicita lo que se entiende por un buen desempeño docente en Chile, sino que además sirve de base para la evaluación que se realiza anualmente a muchos profesores en ejercicio, entonces pareciera claro que hay ciertas debilidades en la formación docente. Sin embargo, también es importante considerar que las dificultades de los primeros años de inserción laboral también se explican, en parte, por la pérdida paulatina de la capacidad de innovación y la pronta adscripción a las prácticas tradicionales de enseñanza (Imbernon, 1994), que por lo demás fueron conocidas por los profesores principiantes desde su rol de alumnos de una escuela. 
Frente a estos resultados, y considerando que la formación de profesores es un tema de política pública porque impacta en la calidad de la educación, surgen dos preguntas: ¿Cómo se hacen cargo las instituciones formadoras de las debilidades de la formación? ¿Cómo desarrollan la autonomía y la capacidad para enfrentarse a contextos específicos?

La segunda área de problemas está referida a la multiplicidad de tareas que deben realizar los profesores desde el primer año de trabajo y está relacionada con las condiciones que las instituciones educativas ofrecen a los nuevos profesores en el período de inserción a la vida profesional.

Al respecto, la mayoría de los profesores novatos dice no tener tiempo para planificar, revisar trabajos y evaluaciones, esto debido al exceso de tareas extra aula que realizan en horas que no son de docencia: realizar turnos de recreo o almuerzo, reemplazos de colegas, participación en eventos propios del centro educativo como celebraciones, actos litúrgicos y otros eventos, además de llevar el registro de notas, realizar informes o entrevistas a apoderados, todo lo cual les impide tener las planificaciones diarias y mantener el trabajo al día, pese a que continúan con el trabajo en sus casas.

Esta multiplicidad de tareas también se da al interior del aula y afecta el tiempo real de aprendizaje, según lo manifestado por los profesores novatos. Por ejemplo, es necesario pasar lista, revisar materiales y tareas, atender situaciones emergentes y dar respuesta a solicitudes externas al aula, todo al mismo tiempo, mientras conducen procesos de enseñanza aprendizaje. Estos resultados llevan a otras dos interrogantes: ¿Cómo podría la institución crear condiciones para apoyar al profesor novato en la primera etapa de vida profesional? ¿Cómo la institución educativa puede apoyar el conocimiento de la escuela, de manera tal que el profesor principiante pueda desarrollarse profesionalmente en ese contexto?

Frente a estos hallazgos parece necesario buscar estrategias innovadoras que contribuyan al desarrollo de competencias complejas como son la toma de decisiones, la reflexión y la colaboración como forma de apoyo para aminorar los problemas de los profesores jóvenes en sus primeros años de inserción a la vida profesional.

En este contexto, es imprescindible que tanto las instituciones formadoras como las instituciones educativas en las que se inician profesionalmente los educadores se hagan cargo del apoyo a los profesores jóvenes a través de procesos de acompañamiento especializado o mentorías, que abarquen tanto el período de práctica como los primeros años de trabajo. De esta manera se contribuiría al desarrollo profesional de los profesores, los instalaría con mejores condiciones para enseñar y evitaría el abandono de la profesión docente en forma prematura.

Lo anterior, permite proyectar la necesidad de diseñar, implementar, aplicar y evaluar procesos de mentoría y formación de mentores, tanto en la universidad como en los centros educativos. Esto concuerda con ideas planteadas por la OCDE, ya en el año 2005, al señalar que "las etapas de formación inicial, inserción y desarrollo profesional deberían de estar mucho más interrelacionadas para crear un aprendizaje coherente y un sistema de desarrollo para los profesores" (OCDE, 2005: 13).

\section{REFERENCIAS BIBLIOGRÁFICAS}

Alen, B. (2009). El acompañamiento de los maestros y profesores en su primer puesto de trabajo.

Profesorado. Revista de curriculum y formación del profesorado, 13 (1), 79-87.

Alliaud, A. (2004). La experiencia escolar de maestros "Inexpertos". Biografías, trayectorias y 
práctica profesional. Revista Iberoamericana de Educación, 34 (3), 1-11.

Alliaud, A. y Antelo, E. (2009). Iniciarse a la docencia. Los gajes del oficio de enseñar. Profesorado. Revista de curriculum y formación del profesorado, 13 (1), 89-100.

Ávalos, B., Carlson, B. y Aylwin, P. (2004). La inserción de profesores neófitos en el sistema educativo: ¿Cuánto sienten que saben y cómo perciben su capacidad docente en relación con las tareas de enseñanza asignadas? Informe proyecto Fondecyt 1020218.

Boerr, I. (2014). El largo camino de convertirse en profesor. Docencia, 54, 52-63.

Cisternas, T. (2011). Dificultades experimentadas por profesores principiantes de Educación Básica en sus prácticas de enseñanza y su relación con la Formación Inicial. Santiago: CIDE / Universidad Alberto Hurtado / CEPPE. Disponible en Internet en www.cide.cl/investigacion/ investigacion_desarrollo_detalle_ceppe.php. Recuperado enero 2015.

Cochran-Smith, M. (2004). Stayers, Leavers, Lovers, and Dreamers: Insight about teacher retention. Journal of Teacher Education, 55 (5), 387-392.

Contreras, I., Vásquez, N., Rittershaussen, S., Solís, M. C., Núñez, C. (2013). La conducción de procesos de enseñanza aprendizaje de profesores novatos: ¿a mayores y mejores oportunidades de práctica en la formación inicial, mejores noveles de desempeño? Informe Proyecto Fondecyt 1100771.

Davis, B. \& Higdona, K. (2008). The Effects of Mentoring/Induction Support on Beginning Teachers' Practices in Early Elementary Classrooms (K-3). Journal of Research in Childhood Education, 22 (3), 261-274.

Díaz, C. y Bastías, C. (2013). Los procesos de mentoría en la formación inicial docente. Rev. Int. Investig. Cienc. Soc., 9 (2), 301-315.

Eirín, R., García, H. M. y Montero, L. (2009). Profesores principiantes e iniciación profesional: un estudio exploratorio. Profesorado. Revista de curriculum y formación del profesorado, 13 (1), 101-115.

Flores, C. (2014). Inducción de profesores novatos en Chile: un estudio de caso. Pensamiento Educativo. Revista de Investigación Educacional Latinoamericana, 51 (2), 41-55.

Feiman-Nemser, S. (2000). From preparation to practice, designing a continuum to strengthen and sustain teaching. Teachers College Record, 103 (6), 1013-1055.

Huberman, M., Gonauer, M. M. \& Marti, J. (1993). The Lives of Teachers. New York: Teachers College.

Imbernon, F. (1994). La formación y el desarrollo profesional del profesorado: hacia una nueva cultura profesional. Barcelona: Grao.

Ingersoll, R. \& Strong, M. (2011). The Impact of Induction and Mentoring Programs for Beginning Teachers: A Critical Review of the Research. Review of Educational Research, 81 (2), 201-233.

Marcelo, C. (2008). El profesorado principiante: Inserción a la docencia. Barcelona: Octaedro.

Marcelo, C. (2009). Los comienzos de la docencia: un profesorado con buenos principios. Profesorado. Revista de curriculum y formación del profesorado, 13 (1), 1-25.

Ministerio de Educación. (2008). Marco para la Buena Enseñanza. Santiago de Chile: MINEDUC.

OCDE. (2005). Teachers matter: attracting, developing and retaining effective teachers. Paris: OCDE

Rodríguez, E. (2007). Las instituciones también cuentan. Los profesores principiantes en educación media frente a los desafíos del desarrollo profesional. Docencia, 33, 64-73.

Ruffinelli, A. (2013). La calidad de la formación inicial docente en Chile: la perspectiva de los profesores principiantes. Calidad en la educación, 39, pp. 117-154.

Smith, T. \& Ingersoll, R. (2004). What Are the Effects of Induction and Mentoring on Beginning Teacher Turnover? American Educational Research Journal, 41 (3), 681-714.

St. Vincent, L. y Gervais, C. (2010). ¿Está preparado el profesor novicio a enfrentar un problema ético en la escuela? Ponencia presentada en el II Congreso Internacional sobre Profesorado Principiante e Inserción Profesional a la Docencia. http://www.crifpe.ca/conferences/view/77. Recuperado enero de 2015. 
Thompson, J. (2007). The first year teacher's survival guide. San Francisco: Jossey-Bass.

Veenman, S. (1984). Perceived problems of beginning teachers. Review of Educational Research, 54 (2), 143-178.

Vonk, J. H. C. (1996). A Knowledge Base for Mentors of Beginning Teachers: Results of a Dutch Experience. In R. McBridge (Ed.), Teacher Education Policy (pp. 112-134). London: Falmer Press. 
